\title{
Research on Special Financial Bonds Strategy of Bio-pharmaceutical Industry in Yunnan
}

\author{
Shuyu Peng
}

Oxbridge College,Kunming University of Science and Technology, 650106

\begin{abstract}
Yunnan bio-pharmaceutical industry is one of the important industries of Yunnan economy, whichdeveloped rapidly. However, bio-pharmaceutical industry in Yunnan provinceis also faced with serious financial difficulties. This paper analyzes the necessity of special financial bond strategy of Yunnan biological pharmaceutical industry and key matters of its implementationin order to offer the reference to the related researchers. KEYWORDS:financial bonds strategy; bio-pharmaceutical industry in Yunnan
\end{abstract}

\section{INTRODUCTION}

As a national fostering industry and a new strategic emerging industry, bio-pharmaceutical industry has made a rapid development in recent years. Bio-pharmaceutical industry has entered a golden period of accelerated development. Statistics show that more and more use of the biological technology has been developed in different extent. New bio-pharmaceutical medicinehas the broad market in recent years in the global innovation drugs listed.

Yunnan has good biological resource endowment, variety, and wide distribution, with $60 \%$ of the country's medicinal animals, plants, natural medicine. Reserves of Yunnan rank first in our country. It has the congenital advantage to develop bio-industry.At present, Yunnan pharmaceutical industry enterprises with a total of 179, have the exclusive national drug varieties 280, accelerate the development of medicine industry has become many areas seize the commanding heights of the development, optimize the industrial structure, change the development mode of favorable selection. After years of nurturing the development, biological industry has become an important economic industry in Yunnan, the growing scale of the industry. From 2002 to 2012, Yunnan biological medicine industry has maintained an average annual growth rate of more than $25 \%$ of the situation. The total value of biological medicine has reached 41.8 billion Yuan last year.The emergence of a large number of at home and abroad have a higher visibility of the pharmaceutical enterprise and brand, the basic development of the pharmaceutical industry is getting stronger, the continuous optimization of the structure, supporting system more and more perfect. Yunnan biological medicine industry are significantly different from other regions of the country, most potential and feature of the place is Yunnan's rich natural medicine resources, national medicine resource advantage, with the policy environment and the improvement of investment environment. Yunnan biomedical industry will become the focus of domestic enterprises, foreign enterprises and other investment institutions in Yunnan. 


\section{Financial Distress of Bio-Pharmaceutical Industry in Yunnan}

\section{A: Less Financing}

Bio-pharmaceutical is divided into three stages.The most important is in the development stage. But as Yunnan pharmaceutical industry started relatively late, early development of the low innovation ability and intellectual property protection of defective make Yunnan biopharmaceutical industry ignored the bio pharmaceutical, only pay attention to the mid late production and sales. From the financial index, the whole of Yunnan biopharmaceutical industry listing Corporation research and development investment only hovering in the total sales of around 5\%, compared with foreign 10\%-20\% investment, the Yunnan research and development investment proportion is quite small. At the same time, without the need for investment in research and development too much money, Yunnan biological pharmaceutical enterprises free funds are sufficient, in terms of solvency and capital structure is generally better than the whole market.

\section{B: High Risk}

The high risk of Yunnan bio-pharmaceutical industry is market risk rather than research and development risk.The lack of innovation has resulted in that the biopharmaceutical industry generally ignores research and development, but pays attention to production and sales in Yunnan. Because of the lack of research and development foundation, once the marketing problems, the enterprise is very easy to fall into financial difficulties. While the sales and advertising activities of biopharmaceutical products in Yunnan and is strictly controlled, single sales channels, sales personnel are very lacking. In addition, due to the same disease biopharmaceutical products many, similar to intense competition, enterprises in developing new products must be quickly into the market, into profits, or face the danger of being eliminated and replaced. These have increased the Yunnan biological pharmaceutical industry market risk. Despite the high risk facing Yunnan bio pharmaceutical industry is not in research and development and in the sales, but it can also bring about the high risk. From the financial indicators point of view, Yunnan biological pharmaceutical industry wool interest rate basically stable at around $36 \%$ and higher than that of the whole market which is about $23 \%$ of the gross margin, fully shows the higher profitability of the bio pharmaceutical industry. Cash flow, cash flow from investment activities of bio pharmaceutical industry, the net outflow volume becomes smaller. This is because of the high market risk industries, although the capital Yunnan biological pharmaceutical enterprises were sufficient, but in foreign investment is also more cautious.

\section{C: Long Turnover Period}

Because of the lack of the research and development stage, sales from the pharmaceutical technology to produce to buy, the whole process is much faster than abroad in Yunnan. Yunnan biological pharmaceutical industry does not have long cycle characteristics. But the bio pharmaceutical industry is different from the general production and circulation industry, because the drug has its own characteristics and long shelf life, coupled with the fierce market competition, the biopharmaceutical industry inventory is more serious than other industries hoarding phenomenon, so Yunnan biological pharmaceutical enterprise inventory turnover is slow. From the financial index, average inventory of Yunnan biopharmaceutical industry turnover rate is only about 4 times a year. The market average inventory turnover rate 
compared too far. Financial Bonds Strategy of Bio-Pharmaceutical Industry in Yunnan

Whether the utility of financial bonds of bio-pharmaceutical industry in Yunnanfalls to real point, the key lies in whether the funds raised by bank is exclusive. As a result of the issue of cost is higher, if the lack of supervision, funds may be diverted to the local government financing platform, the real estate industry and other higher rate of return on investment business. Before the corresponding regulatory measures have not been announced, regulators should supervise from the following aspects:

\section{A: Establish Strict Access System}

At present, the provisions issued by the commitment are a necessity of applying for the issuance of Yunnan biological pharmaceutical industry financial bonds of commercial banks. High cost issue financial bonds issued in the bio-pharmaceutical industry in Yunnan Province can balance the financing of high cost and small micro loans at high interest rates, according to market economy rule in the name of social responsibility requires commercial banks. If the risk of commercial bank profit cannot cover the bear, there is no reason and power to provide small business financial services. In order to solve this problem, a reasonable approach should be given to the implementation of the government of Yunnan province in the biopharmaceutical industry loans of commercial banks must or tax return, to make commercial bank's financing costs, and encourage commercial banks in Yunnan province and the bio pharmaceutical industry to share the preferential policies. The examination and approval of application for bank combined with regulators of bio pharmaceutical industry in Yunnan province development of credit business, the construction of the internal control system and strategic location, and will apply for
Bank of bio pharmaceutical industry in Yunnan province credit scale, future development planning, enterprise customer structure contained in the scope of the study in the examination and approval.

\section{B: Establish Real-time Inspection Mechanism}

The first thing of establish real-time inspection mechanism is strengthen the information disclosure norm further. For its operating conditions, raise funds usage, small micro risk situation of the development of financial business and bonds also cashing regularly should disclose to regulators timely. Secondly, regulators should through the regular and periodic on-site inspection, the use of funds to fully grasp the progress of Yunnan biological pharmaceutical industry financial debt and Yunnan biological pharmaceutical industry loans, strictly control the flow of funds rising. When necessary, the use of funds supervision of audit and inspection department may entrust the third party intermediary for the bio pharmaceutical industry in Yunnan Province financial debt. Finally, according to the "Regulations" issued the measures for the administration of the national interbank bond market to issue financial bonds, Yunnan biological pharmaceutical industry financial bonds of commercial banks should publish to the society regularly use to raise funds, through the strengthening of external debt market discipline effect, urge the bank improve risk management, improve the quality of loan in Yunnan province of biological pharmacy industry, reduce the default risk. Through these measures, the supervision department will realize the financial bonds of it earmarking.

\section{C: Establish Rigorous Punishment Rules}

Regulators should build information filesbased on the situation of foreign information disclosure site inspection and the 
issuance of bonds will be issued for use in Yunnan. The financial institutions to issue Yunnan biological pharmaceutical industry financial debt are almost commercial banks. Finance Leasing Company in the small micro financial market performance of financial institutions active participation into bonds subject to restriction threshold, relaxation of small and medium-sized financial institutions into the bond market financing, provide reasonable capital for small and medium-sized financial institutions supplementary channel. Those who are not in accordance with the requirements of earmarking, free to change the flow of capital, regulatory departments should veto its market access, and reduce the regulatory rating. Through the mechanism of severe punishment, regulators guarantee loans for bio-pharmaceutical industry used effectively.

\section{Conclusions}

Yunnan biological pharmaceutical industry performance for financing atrophy in the cash flow is almost negative to repay the debt. The industry enterprises generally adopt contracted investment policy and the funds will be invested to expand market share. In Yunnan, the biopharmaceutical industry in general to take the way credit, and credit ratio than other industry, which led to the recovery of accounts receivable should speed the relatively slowly. Compared with 25 times of the whole market average annual turnover, the turnover frequency of 5 times a year of biological pharmaceutical industry appears very slow. Yunnan province has established a comprehensive, scientific evaluation system to ensure access, through the issuance of the approval of the bank in the business situation, strategic planning and other aspects of the bio pharmaceutical industry in Yunnan Province financial bonds with high adaptability. It will banish the unstandardized andunsuitable enterprises from the financial bonds.

\section{REFERENCE:}

[1] Hu Ji. A Study on Financial Distress Prediction of Bio-Pharmaceutical Industry inChinaBasedonListedCompanies'FinancialIndicators[ D].Southern University of Finance and Economics, 2009.

[2] Liu Tiantian. The Study on the Cost-benefit of SMEs'Financial Bonds Issued by Commercial Banks[D].Anhui University, 2014.

[3] Deng Yu, An Huaxuan. Survey and Research of Innovation Ability of Enterprisesof Bio-Pharmaceutical Industry in Yunnan [J].Yunnan Management of Science and Technology, 2009, 02.

[4] Cuthbertson K, Nitzsche D. Quantitative financial economics: stocks, bonds and foreign exchange[M]. John Wiley \& Sons, 2005.

[5] Saunders A, Cornett M M. Financial markets and institutions[M]. McGraw-Hill/Irwin, 2012.

[6] van Loon P, Cairns A J G, McNeil A, et al. Modelling the Liquidity Premium on Corporate Bonds[J]. 2014.

[7] Gürtler M, Hibbeln M, Winkelvos C. The impact of the financial crisis and natural catastrophes on CAT bonds[J]. Journal of Risk and Insurance, 2014. 